\title{
Drug Accountability Assessment Original Result
}

National Cancer Institute

\section{Source}

National Cancer Institute. Drug Accountability Assessment Original Result. NCI

Thesaurus. Code C83102.

The outcome of the drug accountability examination. 